\title{
同神经电针镇痛时兴奋的传入 纤维类别初步观察
}

郡殿华 吴建屏 赵志奇

（中国科学院上海生理矼究所）

以电针刺激支配手术区的神经进行外科手术的方法 (简称同神经镇痛), 已经临床实践证 明对浅层组织的疼痛有较满意的镇痛效果 ${ }^{[1]}$. 在临床应用时, 通常在十五分钟至半小时的诱 导期内, 逐渐增加电针刺激强度, 达到病人能耐受的最大强度后再开始手术. 研究在这种情况 下电针刺激兴奋了那些传入神经纤维, 对了解同神经镇痛的作用原理及进一步提高临床镇痛 效果, 都有一定意义. 本文报告在正常人身上用叠加方法记录电针刺㥢引起的动作电位所得 到的结果.

实验对象为六名年龄 21-44 岁的正常人, 男 4 名, 女 2 名. 实验时, 先用电针仪通过圆形 银片电极在小腿后外侧皮肤表面确定腓肠神经位置 (方法见文献[1]), 然后将一对刺激电极插 人外踝以上 10 厘米左右水平腓肠神经所在位置的皮下, 将一对记录电极插人外棵后下方腓肠 神经所在位置的皮下, 电极间距约 3-5 毫米. 刺激及记录电极均以 32 号针炎针表面涂以绝 缘渿制成, 尖端裸露约 1 毫米. 在插人记录及刺激电极后, 经电极施加方形脉冲刺激, 根据受 试者主诉耐心调整电极位置, 直至以最小电流能引起腓肠神经支配区皮肤有明显向下放射的 麻木感为止, 这样可使刺激或记录电极的尖端尽量靠近神经. 电刺激由定流刺擞器产生, 记录 到的神经动作电位经前级放大器、示波器, 再送到 JSY-1 型医学生物用电子计算机进行叠加, 叠加 400-500 次后的波形用 $x-y$ 记录仪或照相记录.

每次实验均在皮肤外测量刺激与记录电极间的距离, 在计算动作电位传导速度时, 将从刺 教伪迹至动作电位第一个正波峰顶的时间作为传导最快的动作电位的传导时间，对随后出现 的传导较慢的动作电位各波, 因确定正波峰顶往往很困难, 故将从刺激伪迹至各负波峰顶的时 间作为传导时间, 由此算出的传导速度比实际的稍慢 ${ }^{[2]}$. 实验时测得的记录及刺激电极附近 皮肤表面温度为 $25^{\circ}-28.5^{\circ} \mathrm{C}$, 在算出各波的传导速度后, 以皮肤温度 ${ }^{\circ} 5^{\circ} \mathrm{C}$ 为标准, 以每增加 $1^{\circ} \mathrm{C}$, 传导速度增加 2 米/秒的数值 ${ }^{[2]}$ 进行校正.

以强度逐渐增加的单个方波(波宽 0.1 毫秒) 刺激腓肠神经, 当刺电流达到 $0.2-0.9 \mathrm{~mA}$ 时,受试者在外踝后下方皮肤开始产生向下放射的麻木感，我们称这时的刺滶强度为感觉间. 麻木感随刺激强度的增加而增强, 最后在此区出现向下放射的胀痛感觉. 一般在感觉闻或稍 高的刺激强度时开始能记录到动作电位, 随刺激强度的增加, 动作电位逐渐增大, 并相继出现 传导速度较慢的各个成份(图 1).

实验时, 先将刺激强度逐渐增加到受试者在腓肠神经支配区感到明确的向下放射的疼痛 
感觉. 此时的刺激电流值为感觉阈的 6.25-11.5 倍 (平均 7.6 倍). 它们比刚出现痛觉时的刺 激强度为高. 这种刺激强度产生的动作电位图形均由一系列传导速度不等的波形组成(图1)，
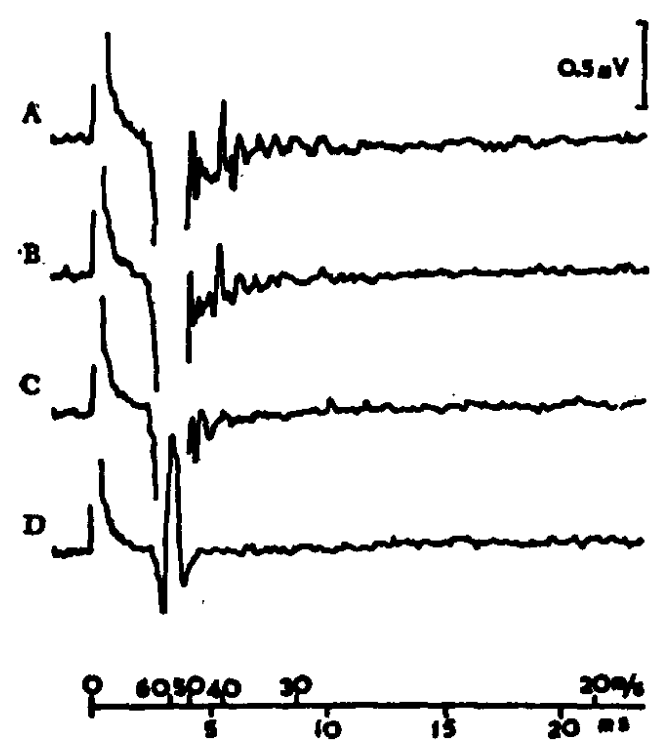

图 1 不同强度的电刺激引起 的腓肠神经动作电位波形

受试者女, 38 岁. 记录 $\mathrm{A}$,刺㠜电流为 5.5 安 安,受试者感受的有明确痛感。记录 B, 剌敞

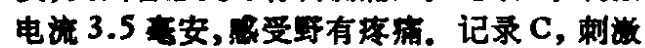

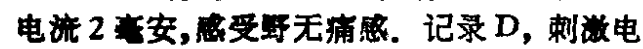
流 1 安安, 爱野无痛感. 这名受试者的最大 电针酎受强度是 1.7 豪安. 图下的标尺表示 经温度校正后的动作电位传导速度及时标。 所有波形均系经叠加 500 次后以 $\mathrm{X}-\mathrm{Y}$ 记录仪 描记的记录

传导最块的动作电位的传导速度为 60.6一70.6 米/秒, 平均 65.3 米/秒. 明确可见的最慢的动作电位的传导 速度为 28.4-38.6 米/秒 (平均 35.5 米/秒). Collins 等 ${ }^{[3]}$ 曾报告以单个电脉冲兴奋人腓肠神经中传导速度 为 25-30 米/秒的 $A \delta$ 纤维时, 病人感到刺痛, 我们观 察的结果和他们是一致的。

记录了有疼痛感觉时的动作电位后，降低刺激强 度, 在几种只引起麻木感的较低的刺激强度下分别记 录动作电位. 然后改用每秒 100 周的重复刺激, 在 15 分钟内逐步增加刺激强度, 直到受试者能耐受的最大 强度为止, 记录这时的刺激电流值（简称最大耐受强 度),参照重复刺激前用相同或相近的刺激电流值的单 刺激所产生的动作电位，以估价受试者能耐受的最强 的重复刺激能兴奋那些传入纤维.

六名受试者的最大耐受强度，都低于引起痛感时 的单刺激的强度. 其中两名受试者的最大耐受强度只 能引起传导最快的动作电位, 传导速度分别为 53.2 米 /秒及 55.5 米/秒, 一名受试者的最大耐受强度产生的 最慢的动作电位的传导速度为 43.7 米/秒, 其余三名 受试者的最大耐受强度 分别为 $0.9 \mathrm{~mA}, 1.6 \mathrm{~mA}, 1.7$ $\mathrm{mA}$, 当时末记录这些强度的单刺激引起的动作电位, 但与之最接近的刺激强度 (分别为 $1 \mathrm{~mA}, 1.8 \mathrm{~mA}$ 及 $2 \mathrm{~mA}$ ) 引起的最慢的动作电位的传导速 度, 分别为 39.1 米/秒, 38.6 米/秒及 40.4 米/秒. 可见, 前两名受试者的最大耐受强度所兴奋 的是传导快的粗纤维, 另四名受试者则兴奋了接近 $A \delta$ 纤维传导速度的较细的传入纤维 (平 均传导速度为 40.4 米/秒), 考虑到用叠加方法记录神经干动作电位方法上的局限性, 就这四 名受试者而言,有一小部分较粗的 $A \delta$ 纤维同时被兴奋, 还是有可能的. 在临床条件下, 手术 时的心理状态, 特别是注射杜冷丁, 可能提高对电针的耐受力. 因此可以推测, 在针麻手术时, 至少有一部分病人能耐受的最大电针刺激强度可能已兴奋相当一部分 $A \delta$ 传人纤维.

临床经验表明, 要得到满意的镇痛效果, 必须利用病人对电针针感的适应, 逐步增加电针 刺激强度, 使病人能耐受更强的电针刺激. 结合本文的结果, 这似乎是提示, 当电针增强到能 兴奋一部分 $A \delta$ 传入纤维时, 可能取得更好的镇痛效果. 动物实验的结果, 也支持这种假设.

总结本文结果, 同神经电针引起向下放射的麻木感时, 主要兴奋了较粗的有䯣鞘纤维, 当 刺激强度达到最大耐受强度时, 可能有部分细的有䯣鞘纤维 $(A \delta)$ 被兴奋.

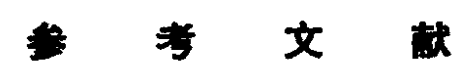

[1] 上海第一人民医院、上海生理研究所针麻协作组，中华外科杂志，1977，1：19一-21.

[2] Buchthal, F. \& Rosenfalck, A., Brain Research., 3 (1966), 1-120.

[ 3 ] Collins, W. F., Nulsen, F. E., Clark, T., Arch. Neurol., 3 (1960), 381-385.

[4]吴建屏、敕志奇、魏仁榆, 中国科学, 1974, 5:526一-533. 